\title{
A Thermo-mechanical cohesive zone model
}

\author{
I. Özdemir · W. A. M. Brekelmans · M. G. D. Geers
}

Received: 1 August 2009 / Accepted: 1 June 2010 / Published online: 27 June 2010

(C) The Author(s) 2010. This article is published with open access at Springerlink.com

\begin{abstract}
In this paper, a cohesive zone formulation that is suitable for the thermo-mechanical analysis of heterogeneous solids and structural systems with contacting/ interacting components, is presented. Well established traction-opening relations are adopted and combined with micromechanically motivated heat flux-opening relations reflecting the evolving heat transfer through the interfaces. The finite element approach for a coupled analysis within an operator-split solution framework is presented and demonstrated with an example problem.
\end{abstract}

Keywords Cohesive zone - Thermomechanics .

Interface element

\section{Introduction}

For heterogeneous materials and multilayered structural systems, in general, interfaces are the weakest links. Knowledge and understanding of thermo-mechanical and physical features and behaviour of interfaces are of utmost importance to improve the performance and reliability of these material systems and engineering structures.

The basic tool for predicting failure of interfaces is linear elastic fracture mechanics (LEFM), which is limited essentially by the bulk constitutive response and the size of the fracture process zone as compared to the crack size.

I. Özdemir · W. A. M. Brekelmans · M. G. D. Geers ( $₫)$

Department of Mechanical Engineering, Eindhoven University of Technology, P.O. Box 513, 5600 MB Eindhoven,

The Netherlands

e-mail: M.G.D.Geers@tue.nl

I. Özdemir

Department of Manufacturing Engineering, Attlım University, Kızılcaşar Mahallesi, İncek, Gölbaşı, 06836 Ankara, Turkey
Furthermore, the LEFM solution cannot capture the real stress distribution at the vicinity of the crack tip. These limitations have been removed by the introduction of the "cohesive zone' concept, which basically removes the crack tip singularity by employing a traction-opening law that reflects the limited strength of the material and the mechanisms of load transfer taking place within the fracture process zone, see Fig. 1. Typically, the interfacial details are not resolved explicitly and the associated load transfer mechanisms are lumped into cohesive zone constitutive relations (tractionopening relations). This approach has been used successfully for the prediction of failure of interfaces subjected to many different boundary conditions, see $[1,2]$ and the cited references therein.

A vast amount of literature exists starting with the seminal work of Xu and Needleman [3], whereby the majority of papers focuses on the mechanical characteristics of the interfaces only. However, for a large number of cases, the actual loading case also includes severe temperature changes (thermal shock) and thermal cycles (thermo-mechanical fatigue) exposed to the system. As discontinuities (cracks) initiate and propagate, they act as barriers for heat flow, therefore affecting the evolving temperature profile within the solid. This clearly influences the thermal strains and may alter the mechanical response significantly in return. Meso-level modeling of concrete failure under fire and the reliability analysis of thermal protection layers are two examples which necessitate a proper thermo-mechanical interface description at different scales as presented in $[4,5]$.

Within the framework of continuum thermodynamics, a thermo-mechanical interface description with damage is presented in [6], though it lacks some physical motivation for certain arguments. Similarly, the authors of [7] present a continuum interface model which is to some extent, independent of the bulk, equipped with its particular thermodynamical 
Fig. 1 Cohesive cracking in different materials, reproduced from [1]
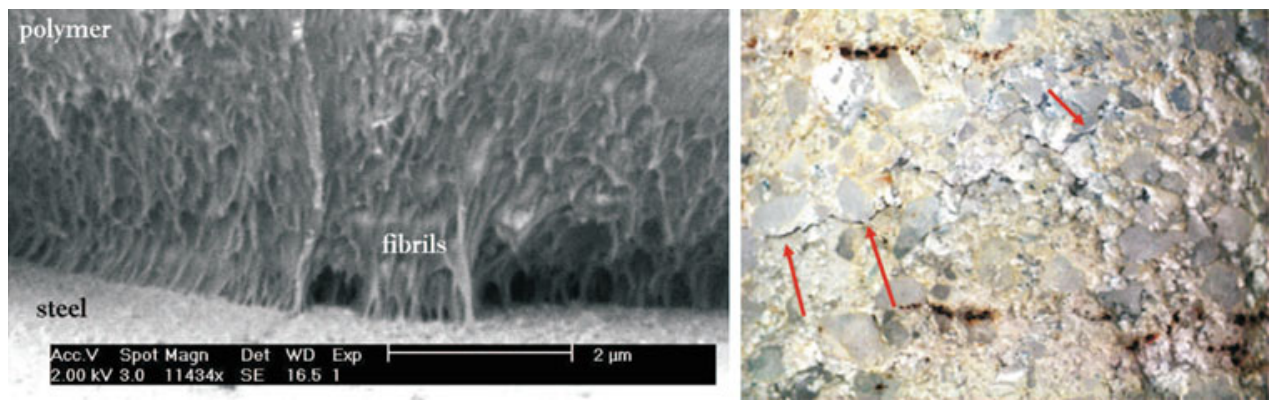

potentials and connected/coupled to the bulk by certain assumptions. Furthermore, it requires some extra effort to convert the formulation into a favorable format considering implementation aspects [8]. Motivated by ductile fracture problems [9] presents a similar formulation in the sense that the interface has its own thermodynamical potentials and an efficient discretization is realized within an X-FEM framework.

Alternatively [10] proposes a micromechanically motivated thermo-mechanical cohesive zone description for fiber-reinforced ceramic matrix composites, which however does not take into account the thermal strains of the bridging fibers. Pursuing a phenomenological approach, the same authors presented a model $[5,11]$ where the heat transfer along the interface is neglected. Though it is sufficient for the loading conditions considered in these studies, in some other cases (e.g. mode II dominated situations), a more rigorous heat flow analysis may become necessary.

Considering idealized load and heat transfer mechanisms suggests to construct the thermal response of all micromechanisms and separating materials involved as the basis for quantifying heat transfer across the cohesive zone. Therefore, the conductivity of the bridging fibers or fibrils and the air within the crack enclosure determines the conductivity attributed to the cohesive zone model, see Fig. 1. Furthermore, thermal expansion of these microstructural components reveals itself as thermal strains in the corresponding traction-opening law and has to be taken into account properly. Moreover, in analogy with the mechanical response, at the crack tip a singularity in the temperature gradient arises [12], which can be effectively handled by introducing the thermal/cohesive zone concept.

It is the aim in the present paper to develop a physically motivated, complete thermo-mechanical cohesive zone model including its finite element formulation, which can be used at the meso-level modeling of heterogenous materials and multilayered structures.

In the next section, the heat transfer across a partially open interface is presented which is the basis for the heat flux-opening relations. The mechanical counter part of the problem is presented in Sect. 3 within a thermo-mechanical context for the sake of completeness. Thereafter, a finite element formulation, which uses the presented interface constitutive laws and a solution algorithm based on the operator-split technique, is briefly summarized. An example problem is elaborated to demonstrate the merits of the presented formulation.

\section{Heat conductance and thermal expansion through an interface}

In case of heat flow, across a partially open cohesive crack, a temperature jump between the two faces of the discontinuity is observed since the coupling between the interfacial load and heat transfer mechanisms are not explicitly resolved in a cohesive zone model. It is important to note that the heat flux through crack bridging matter (e.g. fibrils) has a certain direction which has to be properly accounted for in a coarse scale cohesive zone model.

To this end, the temperature jump is defined as a vectorial quantity according to

$\llbracket \boldsymbol{\theta} \rrbracket=\llbracket \theta \rrbracket \mathbf{m}$

where $\llbracket \theta \rrbracket=\theta^{+}-\theta^{-}$is the temperature difference between the two material points (depicted as + and - ), which were sharing the same position prior to the appearance of the discontinuity and $\mathbf{m}$ is the unit vector directed along the line connecting these two points as shown in Fig. 2. The temperature jump is intimately linked to the heat conduction taking place within the crack, both through the bridging solid parts and air filling the crack enclosure. In fact upon further loading, the crack bridging material gradually disappears and the effective conductance diminishes due to the loss of a heat conducting solid medium. The concept of thermal damage mechanics fits very well to quantify the reduction in effective conductivity, i.e. the heat flow conducted through the interface solid connections/links can be expressed as,

$\mathbf{q}^{s}=-(1-d) k_{s} \llbracket \boldsymbol{\theta} \rrbracket$

where $d$ is the damage variable further discussed in Sect. 3 and $k_{s}$ is an effective thermal conductivity that is largely determined by the conductivity of the crack bridging structures, their fraction within the unit cohesive surface and their 
Fig. 2 Discontinuity splitting a material point

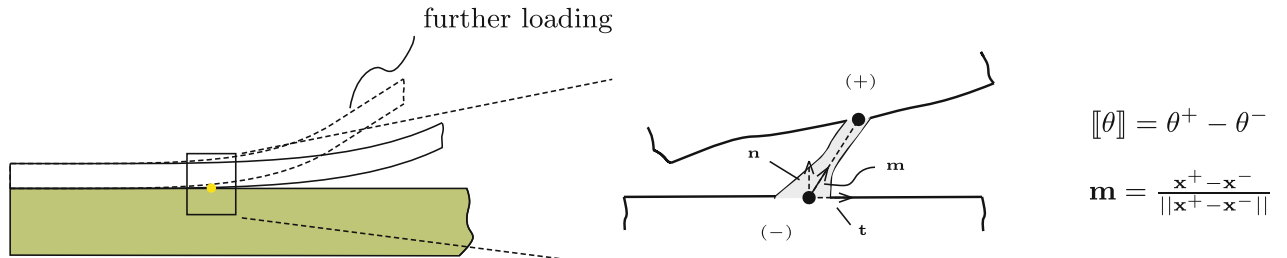

geometric layout. Since cohesive surfaces lack the third dimension (the thickness), $k_{s}$ of Eq. (2), is in fact a heat conductance coefficient, quantifying the heat transported between the two surfaces that is proportional to the conductivity of the bridging solid fractions and inversely proportional with the thickness of the interface. Theoretically, perfectly conducting interfaces should have infinitely large $k_{s}$ values but in a computational setting sufficiently large values should be adopted instead, see Sect. 4.

At this stage, it is more appropriate to decompose the heat flux vector into a normal and a tangential component as,

$q_{n}^{s}=-(1-d) k_{s} \llbracket \theta \rrbracket \mathbf{m} \cdot \mathbf{n}$

$q_{t}^{s}=-(1-d) k_{s} \llbracket \theta \rrbracket \mathbf{m} \cdot \mathbf{t}$

where $\mathbf{n}$ and $\mathbf{t}$ are unit normal and tangent vectors shown in Fig. 2. It is important to note that both components are representing the heat transported from one side to the other side of the cohesive crack. The proposed form for the interfacial heat conduction preserves the geometrical information which can be linked to the current geometry of material crack bridging structures in an average sense.

Heat conducted through air contributes to the normal component of the heat flux. Approximating the temperature difference in normal direction by $\llbracket \theta \rrbracket \mathbf{m} \cdot \mathbf{n}$, heat conducted through air can be expressed as

$q_{n}^{g}=-k_{g} \llbracket \theta \rrbracket \mathbf{m} \cdot \mathbf{n}$.

Additionally, heat is transported also by radiation between the two faces of the crack, but this contribution is very small as reported in [5] and is not taken into account here. Therefore, the normal and tangential components of the interface heat flux can be written as

$$
\begin{aligned}
q_{n} & =-\left((1-d) k_{s}+k_{g}\right) \llbracket \theta \rrbracket \mathbf{m} \cdot \mathbf{n} \\
q_{t} & =-(1-d) k_{s} \llbracket \theta \rrbracket \mathbf{m} \cdot \mathbf{t}
\end{aligned}
$$

where $q_{n}$ and $q_{t}$ are defined as heat fluxes in $\mathbf{n}$ and $\mathbf{t}$ direction, respectively. The magnitude of the interface heat flux which is the heat transported from one side of the crack to the other side is,

$q_{i}=\sqrt{q_{n}^{2}+q_{t}^{2}}$.

In case of load reversal, the crack tends to close but the real contact area is limited by the surface asperities. When the two crack surfaces touch, the real contact localizes at certain spots due to surface roughness. Then the heat flow is constricted to flow through the contacting asperities and the gas in the cavities formed in between them $[5,13]$.

Recently, parallel to the growing interest in multi-scale modelling, improved thermal contact resistance models incorporating complex surface roughness profiles and interaction between the asperities, have been proposed $[14,15]$. Obviously, such models require detailed information about the surface characteristics in order to be used for qualitative predictions. The work of Barber [16], addressing the electrical resistance of contacting elastic rough surfaces, provided lower and upper bounds for electrical contact resistance as a function of surface roughness. Based on a theorem of contact mechanics and assuming that the contacting bodies can be modeled as half spaces, it is proved that there exists a simple relation between the electrical resistance and the incremental elastic compliance of the contacting bodies. The method is exploited in the context of thermal contact resistance of rough elastic surfaces [15] and it is shown that the contact conductivity, $k_{c}$, can be expressed as,

$k_{c}=\frac{1}{M\left(\rho_{1}+\rho_{2}\right)} \frac{d F}{d h}$

where

$\frac{1}{M}=\frac{\left(1-v_{1}\right)}{\mu_{1}}+\frac{\left(1-v_{2}\right)}{\mu_{2}}$

is the composite compliance of the contacting bodies with Poisson's ratio $v_{i}$, shear modulus $\mu_{i}$ and thermal resistance (inverse of thermal conductivity) $\rho_{i}(i=1,2)$. The two bodies are pressed together by the force $\mathrm{F}$ (contact force) and $\mathrm{h}$ is the relative approach (the normal gap). As deduced from Eq. (7), the unit of the contact conductivity is different than that of the bulk since heat flows through the contact area rather than a volume. The contact force $\mathrm{F}$ is strongly dependent on the surface roughness and has a general form,

$F=C g_{N}^{m}$

as derived from fractal geometry based contact models [17]. In Eq. (9), $C$ is the interface compressive stiffness and $g_{N}$ is the normalized gap. The parameter $m$ depends on the statistical character of the asperities (e.g. height) and ranges between 2 and 3.33 for metals. Adopting the given definition 
for $\mathrm{F}$ and using $\mu=\frac{E}{2(1+v)}, k_{c}$ can be expressed as,

$k_{c}=\left[\frac{2}{E_{1}}\left(1-v_{1}^{2}\right)+\frac{2}{E_{2}}\left(1-v_{2}^{2}\right)\right] \frac{1}{\rho_{1}+\rho_{2}} C m g_{N}^{(m-1)}$

by using Eqs. (7), (8) and (9).

For a partially cracked surface, in case of crack closure, heat will be conducted through the undamaged bridging solid fractions and through the contacting asperities over the regions where the two surfaces are completely detached. Assuming that these two heat conduction mechanisms act in parallel, when the crack closes and contact occurs, the interface conductivity, $k_{\text {int }}$, can be expressed as,

$k_{\text {int }}=(1-d) k_{s}+d k_{c}$

where $d$ is the damage variable introduced in Eq. (2) and further discussed in Sect. 3. Assuming that, the contact tangential openings are not significant (in other words $\mathbf{m}$ and $\mathbf{t}$ are supposed to be mutually perpendicular), the components of the interface heat flow vector are defined as,

$$
\begin{aligned}
q_{n, c} & =-k_{\text {int }} \llbracket \theta \rrbracket \mathbf{m} \cdot \mathbf{n} \\
q_{t, c} & =0 .
\end{aligned}
$$

Therefore, in case of crack closure after full separation $(d=1)$, the interface conductivity is merely dictated by the thermal contact conductivity. A closer look to Eq. (10) reveals that, except for incompressible materials, the value of $\left(1-v^{2}\right)$ is close to unity. Furthermore, in most of the cases the modulus of elasticity for the contacting bodies and the compressive stiffness of the interface (or the penalty parameter) are of the same order of magnitude. Therefore for typical values of $m$, and $g_{N}$ values as recorded in the analysis, thermal contact conductivity reaches only a very small fraction of $\frac{1}{\rho_{1}+\rho_{2}}$.

In Fig. 3, an idealized situation, with a cohesive crack and a bridging fiber is shown. It is assumed that the fiber is free to expand or contract without any mechanical constraints and a temperature difference is applied to the system leading to the deformed configuration as shown in Fig. 3. Due to thermal expansion and temperature difference, the fiber elongates or shortens, which results in an increment/decrement in the normal and tangential openings. Since fibers or other crack bridging structures are not explicitly resolved in a cohesive zone approximation of the interface, a correction needs to

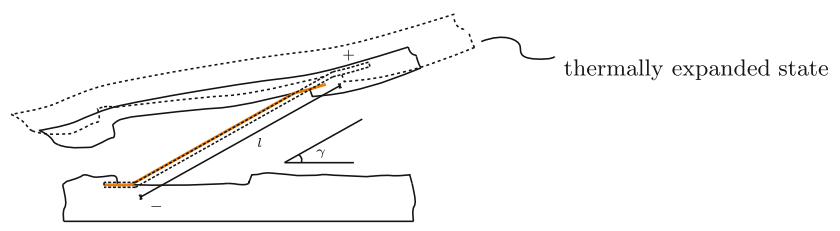

Fig. 3 Free thermal expansion of a bridging fiber be made to account for the interfacial opening as a result of thermal expansion effects in the (constrained) interface. To this purpose, 'temperature jump openings' are introduced, discriminating the openings due to mechanical and thermal loading. Focusing on the deformed geometry and assuming a linear temperature profile along the fiber, when the temperature of the positive side is increased by $\llbracket \theta \rrbracket$, one can write for a single fiber:

$0.5 \alpha \llbracket \theta \rrbracket l \sin \gamma=\Delta_{n}^{T}$

$0.5 \alpha \llbracket \theta \rrbracket l \cos \gamma=\Delta_{t}^{T}$

where $\alpha$ is the coefficient of thermal expansion (CTE) of the fiber, $\llbracket \theta \rrbracket$ is the temperature difference, $\gamma$ is the angle shown in figure, and $\Delta_{n}^{T}$ and $\Delta_{t}^{T}$ are normal and tangential openings due to the temperature jump, respectively.

For the cohesive zone description, $l \sin \gamma$ and $l \cos \gamma$ correspond to the current normal and tangential openings, respectively. Since the previous analysis is based on a single fiber only, the CTE of the fiber should be replaced by a more representative value, since the collective response of many crack bridging agents, their geometric arrangement and volumetric fraction influences the openings due to the temperature jump. Therefore in a more general format, Eq. (13a) and (13b), are reformulated as

$\Delta_{n}^{T}=\alpha_{\text {int }} \llbracket \theta \rrbracket \Delta_{n}$

$\Delta_{t}^{T}=\alpha_{\mathrm{int}} \llbracket \theta \rrbracket \Delta_{t}$

where $\alpha_{\text {int }}$ is the CTE of the interface, $\Delta_{n}$ and $\Delta_{t}$ are the current normal and tangential openings, respectively. In [6], the openings due to the temperature jump appear naturally as a result of the general interfacial free energy expression.

In the following section, the thermo-mechanics of a solid separated by an interface is elaborated both in strong and weak form. Thereafter, the presented concepts are recast in a discretized format suitable for application within a finite element framework.

\section{Thermo-mechanics}

In Fig. 4, a body partially separated by a cohesive crack across the internal boundary $\Gamma_{i}$ is shown. In a geometrically nonlinear setting, the mechanical equilibrium in terms of the Cauchy stress tensor $\sigma$ is written as,

$\nabla \cdot \sigma+\mathbf{b}=\mathbf{0}$

where $\nabla$ is the gradient with respect to the current configuration and $\mathbf{b}$ is the body force vector. At the cohesive crack, the traction continuity condition,

$\mathbf{t}^{+}=-\mathbf{t}^{-}$ 
Fig. 4 Solid body partially separated by a cohesive crack and interface continuity conditions

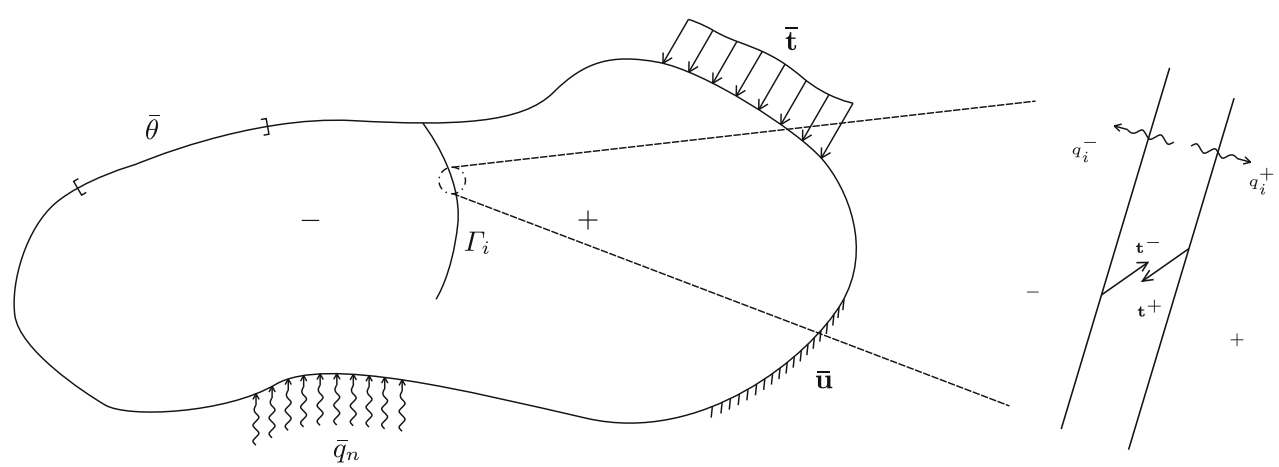

has to be satisfied where the superscripts ${ }^{+}$and ${ }^{-}$indicate approaching to the interface from + and - sides (see Fig. 4) respectively.

In the absence of internal heat sources, the thermal equilibrium in the current configuration is expressed as,

$\rho c_{v} \dot{\theta}+\nabla \cdot \mathbf{q}=0$

where $\rho$ is the density and $c_{v}$ is the heat capacity of the material. Furthermore, taking the inflowing heat as positive, the heat flux vector $\mathbf{q}$ has to satisfy the continuity condition

$q_{i}^{+}=-q_{i}^{-}$

everywhere along the cohesive crack. By applying the Galerkin procedure, the weak forms of the balance equations are obtained as,

$$
\begin{gathered}
\int_{V} \sigma: \nabla \delta \mathbf{u} d V=\int_{V} \mathbf{b} \cdot \delta \mathbf{u} d V+\int_{\Gamma_{t}} \overline{\mathbf{t}} \cdot \delta \mathbf{u} d \Gamma \\
+\int_{\Gamma_{i}^{+}} \mathbf{t}^{+} \cdot \delta \mathbf{u}^{+} d \Gamma+\int_{\Gamma_{i}^{-}} \mathbf{t}^{-} \cdot \delta \mathbf{u}^{-} d \Gamma \\
\int_{V} \rho c_{v} \dot{\theta} \delta \theta d V+\int_{V} \mathbf{q} \cdot \nabla \delta \theta d V=\int_{\Gamma_{q}} \bar{q} \delta \theta d \Gamma \\
+\int_{\Gamma_{i}^{+}} q_{i}^{+} \delta \theta^{+} d \Gamma+\int_{\Gamma_{i}^{-}} q_{i}^{-} \delta \theta^{-} d \Gamma
\end{gathered}
$$

where it is supposed that $\delta \mathbf{u}=\mathbf{0}$ on $\Gamma_{u}$ and $\delta \theta=0$ on $\Gamma_{\theta}$. By using Eq. (16) and (18), Eqs. (19a) and (19b) can be expressed as;

$$
\begin{aligned}
& \int_{V} \sigma: \nabla \delta \mathbf{u} d V=\int_{V} \mathbf{b} \cdot \delta \mathbf{u} d V+\int_{\Gamma_{t}} \overline{\mathbf{t}} \cdot \delta \mathbf{u} d \Gamma \\
&+\int_{\Gamma_{i}^{+}} \mathbf{t}^{+} \cdot\left(\delta \mathbf{u}^{+}-\delta \mathbf{u}^{-}\right) d \Gamma \\
& \int_{V} \rho c_{v} \dot{\theta} \delta \theta d V+\int_{V} \mathbf{q} \cdot \nabla \delta \theta d V=\int_{\Gamma_{q}} \bar{q} \delta \theta d \Gamma \\
&+\int_{\Gamma_{i}^{+}} q_{i}^{+}\left(\delta \theta^{+}-\delta \theta^{-}\right) d \Gamma .
\end{aligned}
$$

Here, $\Gamma_{\bar{t}}$ and $\Gamma_{\bar{q}}$ are the parts of the boundaries where the prescribed traction $(\overline{\mathbf{t}})$ and prescribed normal heat fluxes $(\bar{q})$ are applied. The boundary integrals over $\Gamma_{i}^{+}$in Eqs. (20a) and (20b) are the non-standard extra terms due to the cohesive crack, detailed further hereafter.

Considering a discretization by 2-noded elements for a 2D problem as shown in Fig. 5, the surface integral over $\Gamma_{i}$ in Eq. (20a) is converted into the form,

$$
\int_{\Gamma_{i}^{+}} \mathbf{t}^{+} \cdot\left(\delta \mathbf{u}^{+}-\delta \mathbf{u}^{-}\right) d \Gamma=\sum_{k=1}^{n_{e l}} \int_{\Gamma_{e}^{k}} \underline{t} \delta \underline{\Delta} d \Gamma
$$

where $n_{e l}$ is the number of interface elements in the discretization, $\underline{t}=\left[\begin{array}{ll}t_{n} & t_{t}\end{array}\right]$ and $\delta \underline{\Delta}=\left[\begin{array}{ll}\delta \Delta_{n} & \delta \Delta_{t}\end{array}\right]^{T}$. As shown in Fig. $5, \Delta_{n}$ and $\Delta_{t}$ are defined with respect to the local coordinate system defined on the mid-plane of the interface
Fig. 5 Two noded interface element, undeformed and deformed configurations
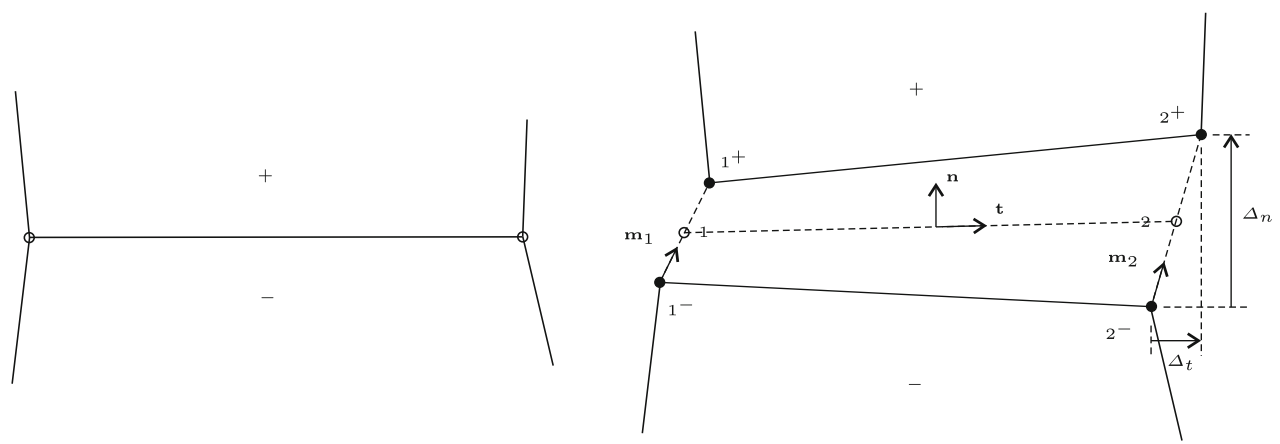

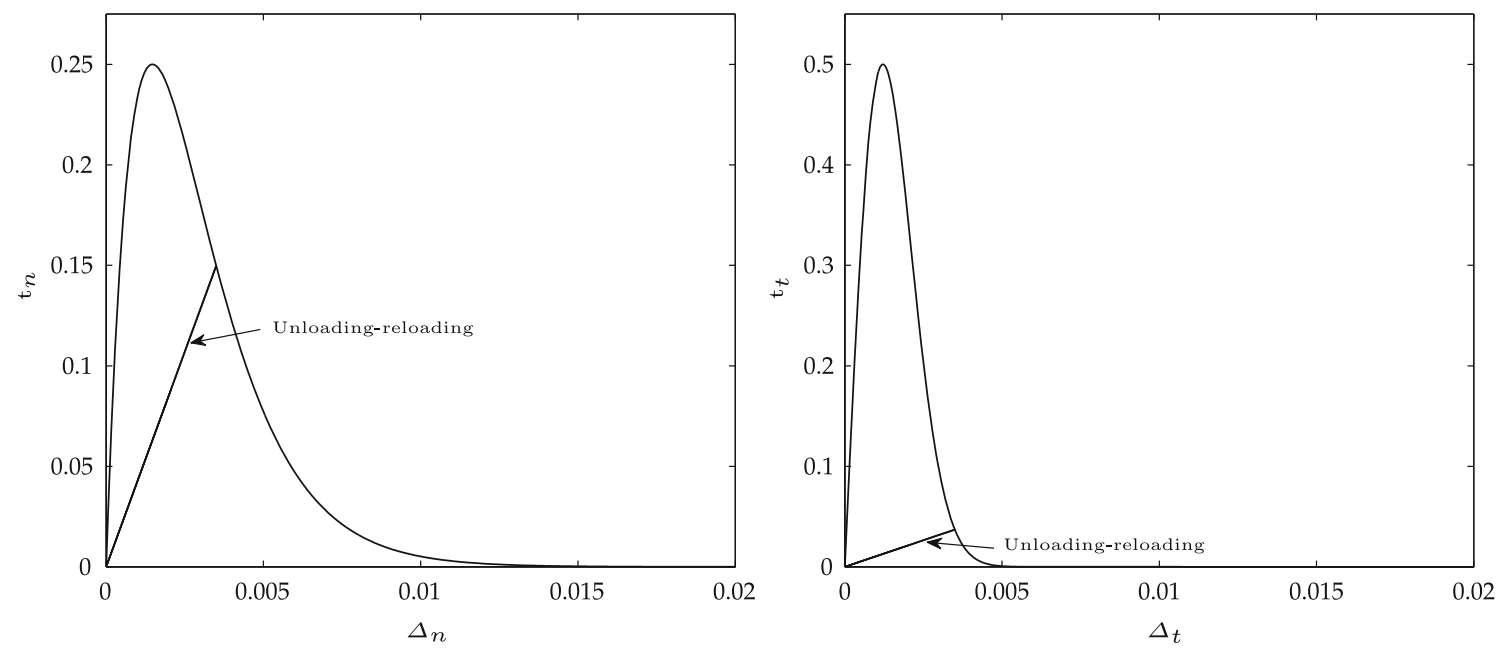

Fig. 6 Normal traction-opening relation (left), tangential traction-opening relation (right)

element. $t_{n}$ and $t_{t}$ are the normal and tangential components of $\mathbf{t}^{+}$with respect to the same local coordinate system. The determination of $t_{n}$ and $t_{t}$ requires constitutive relations in terms of openings, for which the improved $\mathrm{Xu}-\mathrm{Needleman}$ law [23],

$$
\begin{aligned}
t_{n}= & \frac{\phi_{n}}{\delta_{n}}\left(\frac{\Delta_{n, m}}{\delta_{n}}\right) \exp \left(-\frac{\Delta_{n, m}}{\delta_{n}}\right) \exp \left(-\frac{\Delta_{t, m}^{2}}{\delta_{t}^{2}}\right) \\
t_{t}= & 2 \frac{\phi_{t}}{\delta_{t}}\left(\frac{\Delta_{t, m}}{\delta_{t}}\right)\left(1+\frac{\Delta_{n, m}}{\delta_{n}}\right) \\
& \times \exp \left(-\frac{\Delta_{t, m}^{2}}{\delta_{t}^{2}}\right) \exp \left(-\frac{\Delta_{n, m}}{\delta_{n}}\right)
\end{aligned}
$$

is adopted with the proper replacements $\Delta_{n, m}$ and $\Delta_{t, m}$ which are defined as

$$
\begin{aligned}
& \Delta_{n, m}=\Delta_{n}-\Delta_{n}^{T} \\
& \Delta_{t, m}=\Delta_{t}-\Delta_{t}^{T}
\end{aligned}
$$

Traction-opening relations given by Eq. (22), with an unloading-reloading cycle are shown in Fig. 6. The irreversible behaviour is based on a single history parameter $\Delta_{\text {max }}$, representing the maximum effective opening reached during the loading history whereby the effective opening is defined as

$$
\Delta_{\text {eff }}=\sqrt{\beta^{2} \Delta_{t}^{2}+\Delta_{n}^{2}}
$$

where $\beta$ is a scaling parameter. This expression for the effective opening is motivated by considerations on fracture mechanics and propagation of mixed mode cracks [18]. The proposed crack propagation criteria $[19,20]$ has a form where the shear stress components are multiplied by a coefficient which takes a value between 0 and 1 as a function of Poisson's ratio of the medium. Relying on these analytical studies, $\beta$ is taken as 0.5 in this work. Loading takes place when $\Delta_{\text {eff }}=$
$\Delta_{\text {max }}$ and $\dot{\Delta}_{\text {eff }} \geq 0$ and unloading (or reloading) when $\Delta_{\text {eff }}<$ $\Delta_{\text {max }}$. The traction expressions in case of unloading and the corresponding material tangents are given in the Appendix. In case of repeated loadings, the interfacial response weakens as the number of unloading-reloading cycles increases. The traction-opening relations used in the present work can not capture such effects and in case of repeated loadings, dedicated models such as the one presented in [21,22] should be used. In case of crack closure and contact, the normal traction takes the following form,

$t_{n}=C g_{N}^{m}$

based on the fractal models of contact. In this work, $m$ is taken as 2 and $C$ is chosen as a large value depending on the material constants of the problem/system in hand.

The damage parameter $d$ introduced in Eq. (2), is defined as the ratio of $\frac{\Delta_{\max }}{\Delta_{c r}}$ with the critical effective opening,

$\Delta_{c r}=\sqrt{\beta^{2} \Delta_{t, c r}^{2}+\Delta_{n, c r}^{2}}$

where $\Delta_{t, c r}$ and $\Delta_{n, c r}$ are the tangential and normal openings corresponding to small traction values (in this work $0.1 t_{t}^{\max }$ and $0.1 t_{n}^{\max }$ ) in the post-peak regime of the traction opening curves, respectively. It is ensured that, $d \leq 1.0$ in case of excessive openings. The interface integral of Eq. (20b) is expressed as

$\int_{\Gamma_{i}} q_{i}\left(\delta \theta^{+}-\delta \theta^{-}\right) d \Gamma=\sum_{k=1}^{n_{e l}} \int_{\Gamma_{e}^{k}} q_{i} \delta \llbracket \theta \rrbracket d \Gamma$

In a discrete setting, the temperature jump vector introduced in Eq. (1), is expressed as

$$
\begin{aligned}
& \llbracket \boldsymbol{\theta} \rrbracket=N_{1}\left(\theta_{1^{+}}-\theta_{1^{-}}\right) \mathbf{m}_{1}+N_{2}\left(\theta_{2^{+}}-\theta_{2^{-}}\right) \mathbf{m}_{2} \\
& \text { with } \mathbf{m}_{1}=\frac{\mathbf{x}_{1^{+}}-\mathbf{x}_{1^{-}}}{\left\|\mathbf{x}_{1^{+}}-\mathbf{x}_{1^{-}}\right\|} \text {and } \mathbf{m}_{2}=\frac{\mathbf{x}_{2^{+}}-\mathbf{x}_{2^{-}}}{\left\|\mathbf{x}_{2^{+}}-\mathbf{x}_{2^{-}}\right\|}
\end{aligned}
$$


Fig. 7 Thermo-mechanical analysis of a granular microstructure, geometry and boundary conditions

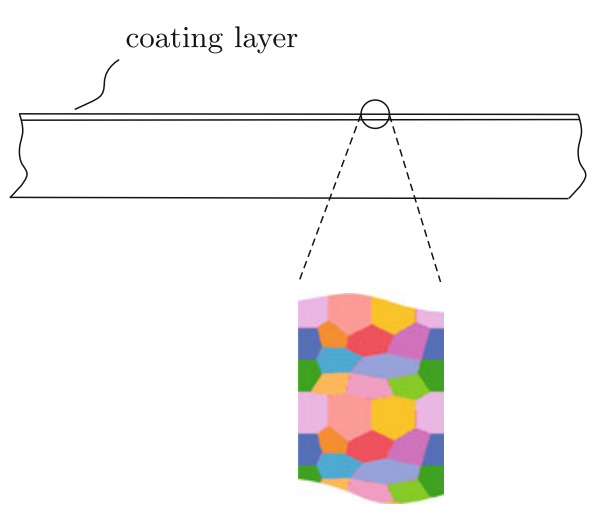

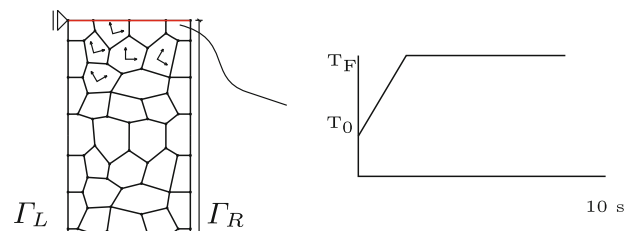

$\Gamma_{L}$ and $\Gamma_{R}$ : periodic displacement b.c.

$\Gamma_{L}$ and $\Gamma_{R}$ : insulated where $N_{1}$ and $N_{2}$ are the standard 1-D shape functions. With this approximation, at a particular integration point $\mathrm{p}$, the normal and tangential components of the interface heat flux vector can be evaluated as

$q_{n}=\left.\left((1.0-d) k_{s}+k_{g}\right) \llbracket \boldsymbol{\theta} \rrbracket\right|_{p} \cdot \mathbf{n}$

$q_{t}=\left.(1.0-d) k_{s} \llbracket \boldsymbol{\theta} \rrbracket\right|_{p} \cdot \mathbf{t}$.

On the basis of Eqs. (6) and (29), the interface heat flux is determined.

The presented element is implemented in a commercial FE software environment and the coupled thermo-mechanical analysis is carried out with a staggered solution scheme. In each load increment, two uncoupled sub-problems, namely the thermal and mechanical equilibrium are solved by the Newton-Raphson method, sequentially. The material tangent operators of the interface elements are presented in the Appendix.

\section{Thermo-mechanical analysis of a granular microstructure}

Due to their high temperature resistance, technical ceramics are used as the base material for specific structural parts and in some cases they are used as coating layers to protect vulnerable components from adverse effects of severe temperature changes. They have a granular microstructure with typical grain dimensions in the micron range or larger. An idealized example case focusing on the thermo-mechanical analysis of such a microstructure is presented in order to investigate the influence of thermo-mechanical cohesive zone description on the evolution of the thermal fields.

A small sub-domain of a coating layer, with the geometry and granular microstructure shown on the right-hand side of Fig. 7, is extracted in order to conduct the thermomechanical analysis. The microstructure is composed of single crystal alumina grains with a tetragonal crystal
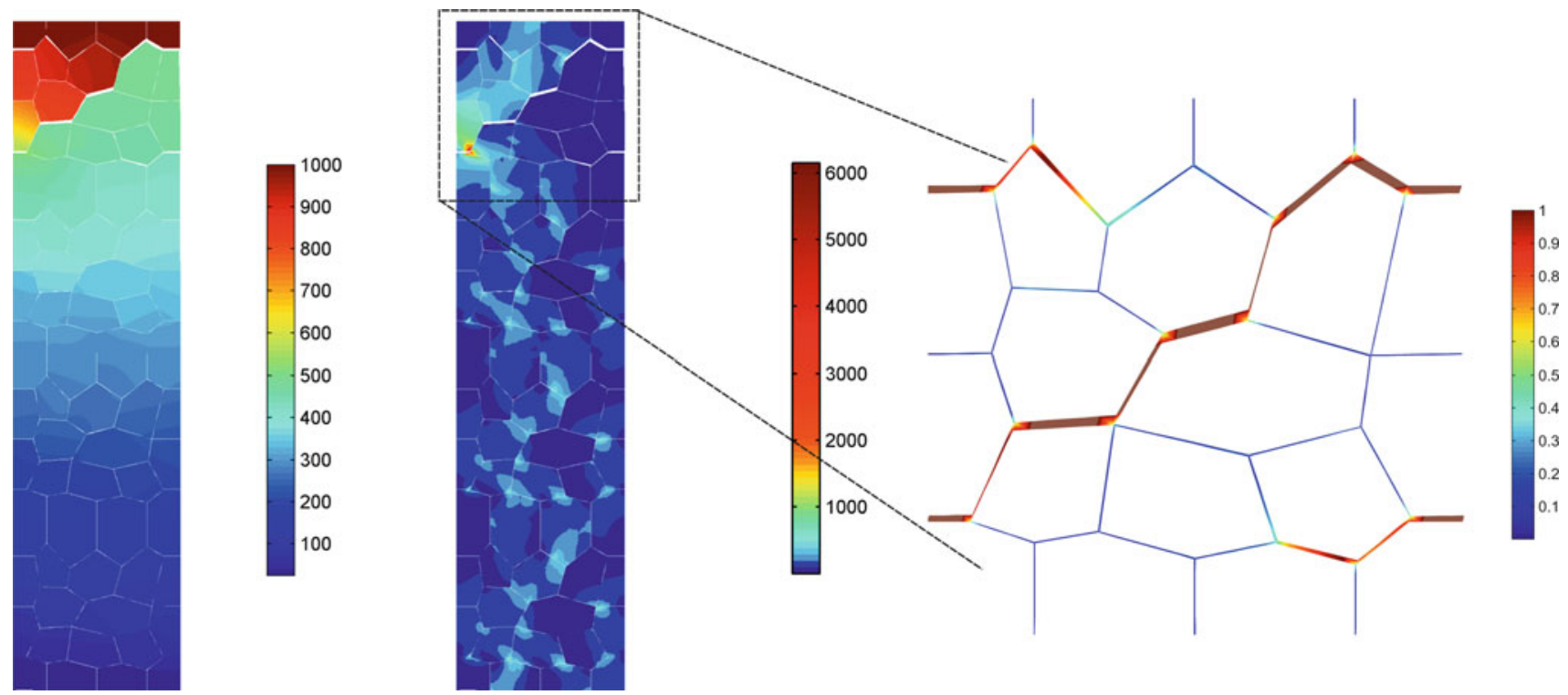

Fig. 8 From left to right: Temperature $\left({ }^{\circ} \mathrm{C}\right)$, magnitude of heat flux $\left(\mathrm{W} / \mathrm{mm}^{2}\right)$, interface damage for low strength interface; $t_{n}^{\max }=100 \mathrm{MPa}, t_{t}^{\max }=$ $200 \mathrm{MPa}$ 

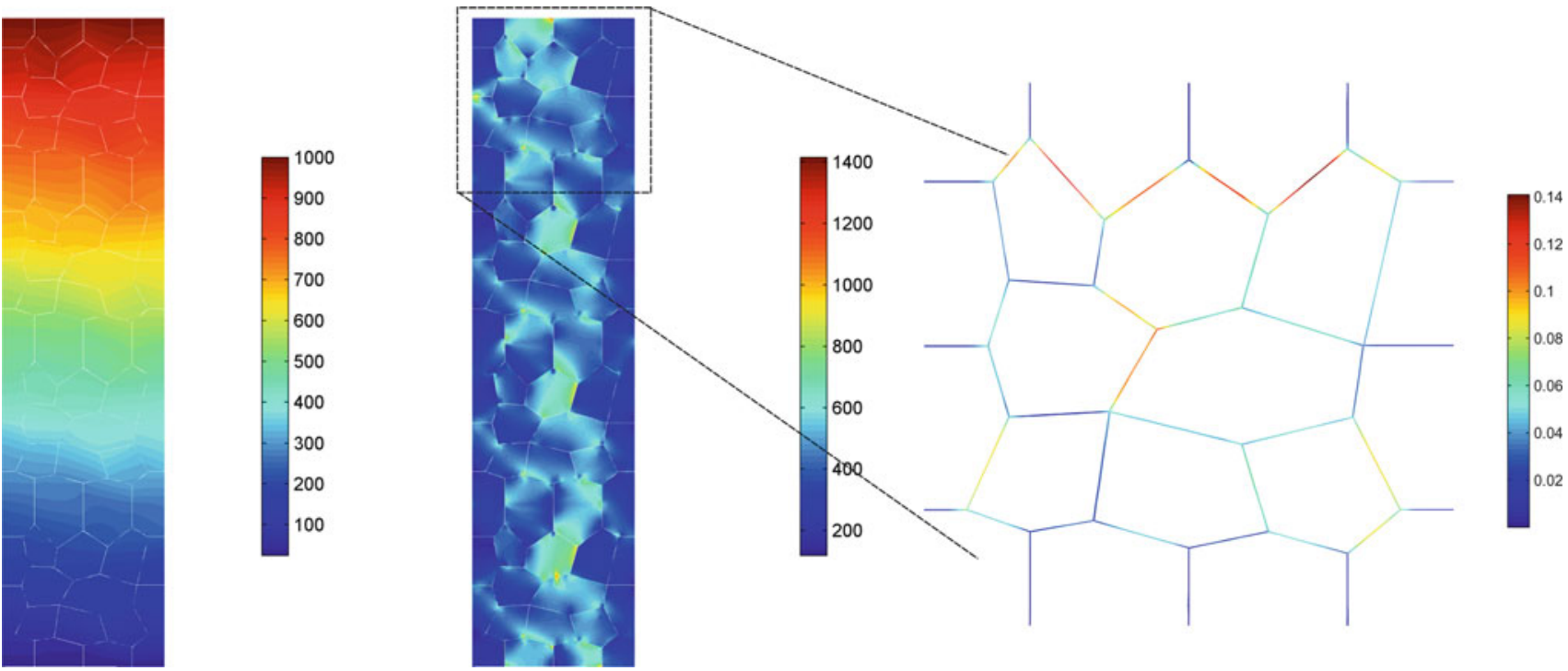

Fig. 9 From left to right: Temperature $\left({ }^{\circ} \mathrm{C}\right)$, magnitude of heat flux $\left(\mathrm{W} / \mathrm{mm}^{2}\right)$, interface damage for high strength interface; $t_{n}^{\max }=800 \mathrm{MPa}, t_{t}^{\max }=$ $1,600 \mathrm{MPa}$

structure. The corresponding anisotropic mechanical constants are taken to be $c_{11}=465 \mathrm{GPa}, c_{22}=465 \mathrm{GPa}$, $c_{33}=563 \mathrm{GPa}, c_{12}=124 \mathrm{GPa}, c_{13}=117 \mathrm{GPa}, c_{44}=$ $233 \mathrm{GPa}$ and the conductivities in the principal directions are given as 38 and $10 \mathrm{~W} / \mathrm{mK}$ [24]. The heat capacity and density values are $c_{v}=1,200 \mathrm{~J} / \mathrm{kg} \mathrm{K}, \rho=2,700 \mathrm{~kg} / \mathrm{m}^{3}$ and the principal values of the anisotropic thermal expansion coefficients are $7.910^{-6} 1 / \mathrm{K}$ and $8.810^{-6} 1 / \mathrm{K}$. The orientation of the principal axis within each alumina grain is taken to be random and the difference between the neighbouring grains are large enough to exclude the presence of a certain texture within the microstructure. Since the contact conductivity model is based on the interaction of isotropic materials, the larger principal conductivity value is used in Eq. (10). The top boundary is exposed to a ramp type prescribed temperature boundary condition which reaches the peak temperature of $1,000^{\circ} \mathrm{C}$ within 4 seconds and which is kept constant until the end of the loading duration of $10 \mathrm{~s}$. The bottom surface is kept at $20^{\circ} \mathrm{C}$ throughout the analysis. Mechanically, periodic boundary conditions are applied on the left and right boundaries as shown in the figure. Plane strain conditions are assumed and the thermo-mechanical
Fig. 10 Temperature profile along the mid-section

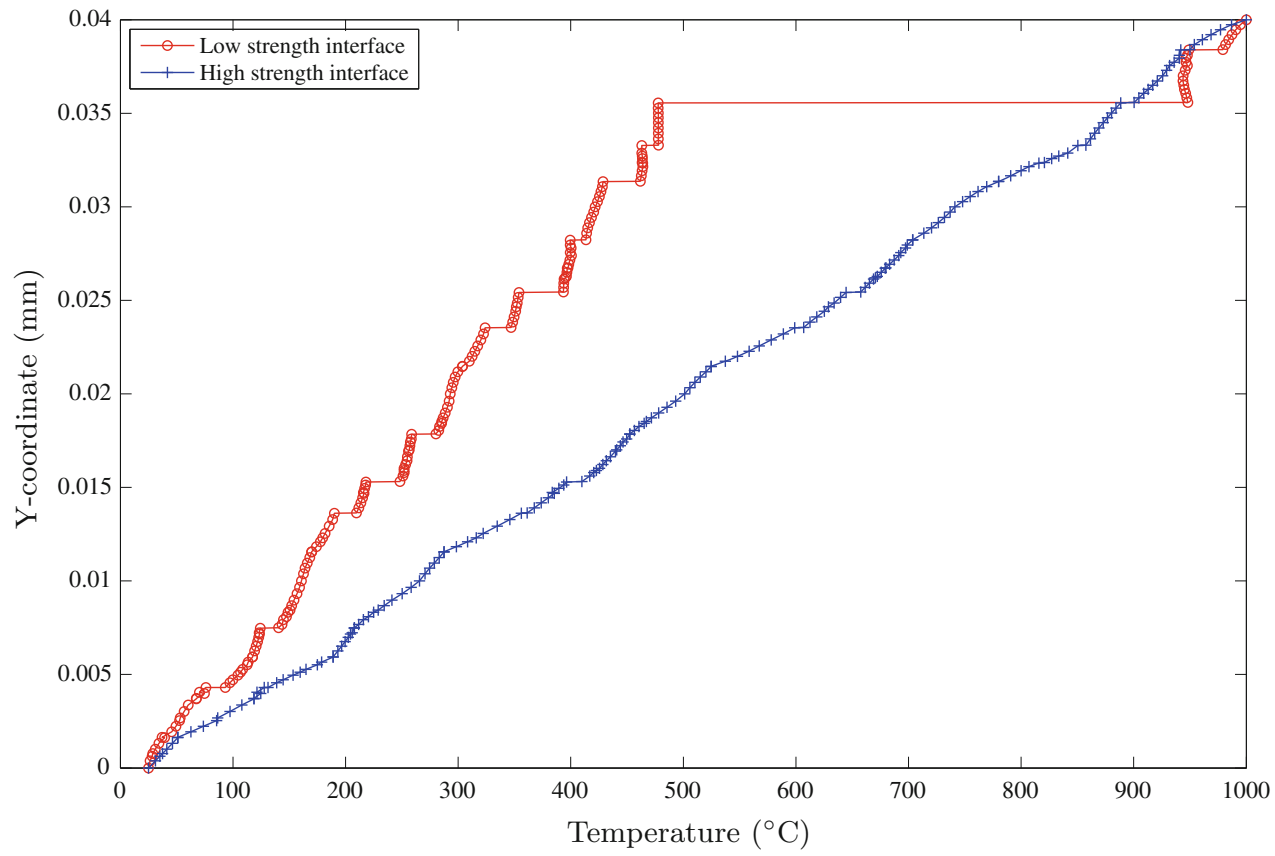


Fig. $11 \sigma_{y y}$ (yy component of Cauchy stress tensor) (MPa) distribution for the case of low strength interface (left) and high strength interface (right)

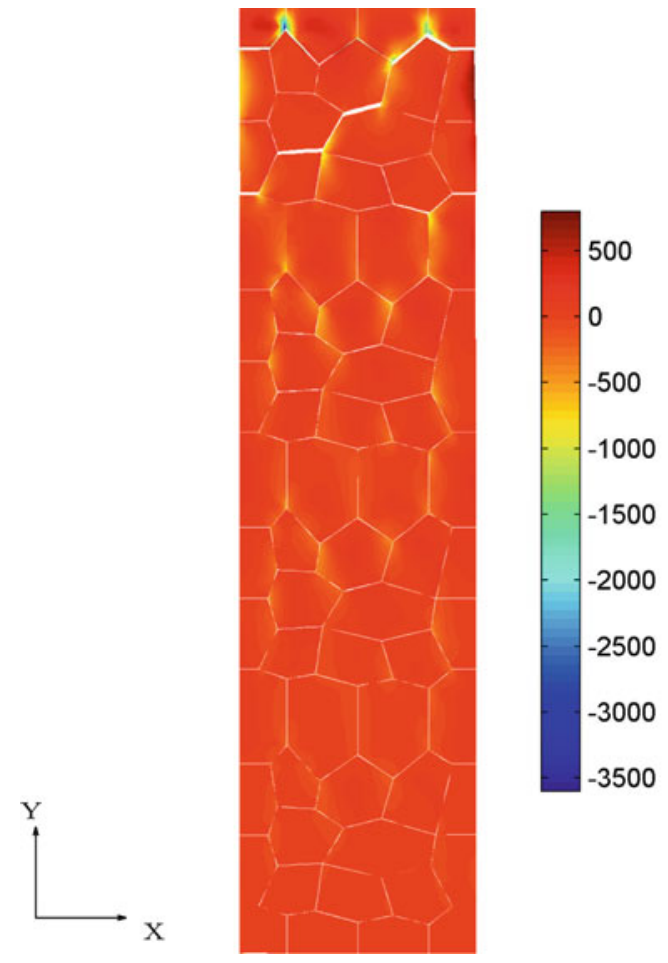

cohesive zone elements are placed along the grain boundaries.

The $k_{s}$ value for the interfaces is determined on the basis of the conductivity values for the bulk material and the characteristic openings for the interfaces calculated from the energies and maximum traction values. Furthermore, in case of mechanically intact interfaces, the influence of $k_{s}$ on the resulting temperature profile should be very small which can be monitored on the basis of temperature jumps across the interfaces. As the $k_{s}$ value is taken larger, the temperature jump diminishes and in the limit of infinitely large $k_{s}$, the temperature jump becomes zero. Therefore some preliminary analysis is carried out to determine the $k_{s}$ values resulting in $k_{s}=5 \cdot 10^{3} \mathrm{~W} / \mathrm{mm}^{2} \mathrm{~K}$. It is also observed that the influences of $\Delta_{n}^{T}$ and $\Delta_{t}^{T}$ are negligibly small therefore interface thermal expansion, $\alpha_{\mathrm{int}}$, is taken to be zero. Keeping the values of $\phi_{n}=40 \mathrm{~J} / \mathrm{m}^{2}$ and $\phi_{t}=80 \mathrm{~J} / \mathrm{m}^{2}$ the same, two different analyses are carried out with $t_{n}^{\max }=100 \mathrm{MPa}, t_{t}^{\max }=200 \mathrm{MPa}$ and $t_{n}^{\max }=800 \mathrm{MPa}, t_{t}^{\max }=1,600 \mathrm{MPa}$, respectively.

These values are comparable with the values given in [25] which focuses on the response of fine grained alumina samples under dynamic mechanical loading. Since the energies are identical, the two interfacial responses are distinguished as 'high strength' and 'low strength' interfaces due to large differences in the maximum traction values $\left(t_{n}^{\max }\right.$ and $\left.t_{t}^{\max }\right)$ used.

In Figs. 8 and 9, the resulting temperature profiles, the magnitude of the heat flux vectors along with the interfacial damage distribution are shown for both cases. As a matter of fact, as the interfacial damage increases, the heat flow through the interface becomes more difficult. Therefore as the interfacial damage evolves, one expects temperature discontinuities as supported by the temperature profiles presented in Fig. 10. In the case of a 'low strength' interface, the deformation localizes and leads to a severe damage profile along a certain path, see Fig. 8. However, in case of a 'high strength interface', the interfacial damage evolves in a spatially distributed pattern as seen in Fig. 9.

Furthermore, the correlation between the damage and the magnitude of the heat flux distribution suggests that as the crack further opens up, heat flow is redirected to more conductive regions. A comparison of heat flow patterns for the two cases clearly shows this effect, as the heat follows the intact path in case of more severe interface damage.

Furthermore, as shown in Fig. 10, in case of strong interface damage, the temperature jumps along the interfaces could reach very significant levels, which in turn will influence the local mechanical response as exemplified in Fig. 11, where the resulting $\sigma_{y y}$ (yy component of the Cauchy stress tensor) profiles are presented. The resulting stress profiles are quite different both qualitatively and quantitatively. Very severe compressive stresses develop due to pressure action between grains typically at juncture points of the polycrystal structure. In case of a 'low strength' interface, as a result of localized deformation, tensile stresses are relieved in the close vicinity of the crack path whereas for a 'high strength' interface there is still significant amount of load transfer across the interfaces throughout the domain. 


\section{Conclusion and outlook}

Motivated by the thermal shock analysis of heterogenous materials, a thermo-mechanical cohesive zone description is presented which is suitable for the analysis of material interfaces at different scales, ranging from grain boundaries to multi-ply structural components. The physical heat transport mechanisms are taken into account within the limitations of a macroscopic cohesive zone formulation. As shown by the example problem, the evolution of mechanical damage might influence the thermal field quantities both qualitatively and quantitatively. As the heat conduction characteristics evolve due to interfacial damage, the heat flow pattern and local stress state might change significantly. Therefore, the proposed formulation assists in acquiring a better understanding of the failure initiation and propagation under severe thermal loading conditions. Furthermore, the efficiency of protective layers in terms of thermal performance can be investigated in a better way and can be optimized. Temperature dependency of the interface parameters, e.g. fracture energies, can be incorporated in the formulation easily provided that these dependencies are known. In conclusion, the thermo-mechanical analysis of material interfaces can be done in an effective way using the proposed formulation.

Open Access This article is distributed under the terms of the Creative Commons Attribution Noncommercial License which permits any noncommercial use, distribution, and reproduction in any medium, provided the original author(s) and source are credited.

\section{Appendix}

The solution of the mechanical and thermal equilibrium equations are conducted within an incremental-iterative framework by means of the Newton-Raphson method. Since the problem is solved by an operator-split technique, incrementally the mechanical and thermal equilibrium equations are solved in an uncoupled way. The material tangent relations of the traction-opening relations, which are necessary for the consistent linearization of the mechanical equilibrium equations are given first.

In case of loading, the derivatives

$$
\begin{aligned}
\frac{\partial t_{n}}{\partial \Delta_{n, m}}= & \frac{\phi_{n}}{\delta_{n}} \exp \left(-\frac{\Delta_{n, m}}{\delta_{n}}\right)\left(\frac{1}{\delta_{n}}-\frac{\Delta_{n, m}}{\delta_{n}^{2}}\right) \exp \left(-\frac{\Delta_{t, m}^{2}}{\delta_{t}^{2}}\right) \\
\frac{\partial t_{n}}{\partial \Delta_{t, m}}= & \frac{\phi_{n}}{\delta_{n}} \exp \left(-\frac{\Delta_{n, m}}{\delta_{n}}\right) \\
& \times\left(-2 \frac{\Delta_{n, m}}{\delta_{n}} \frac{\Delta_{t, m}}{\delta_{t}^{2}} \exp \left(-\frac{\Delta_{t, m}^{2}}{\delta_{t}^{2}}\right)\right)
\end{aligned}
$$

$$
\begin{aligned}
\frac{\partial t_{t}}{\partial \Delta_{t, m}}= & \left(1+\frac{\Delta_{n, m}}{\delta_{n}}\right) \exp \left(-\frac{\Delta_{n, m}}{\delta_{n}}\right) \\
& \times \exp \left(-\frac{\Delta_{t, m}^{2}}{\delta_{t}^{2}}\right) 2 \frac{\phi_{t}}{\delta_{t}^{2}}\left(1-2 \frac{\Delta_{t, m}^{2}}{\delta_{t}^{2}}\right) \\
\frac{\partial t_{t}}{\partial \Delta_{n, m}}= & \frac{\phi_{t}}{\delta_{n}}\left(\frac{\Delta_{n, m}}{\delta_{n}}\right)\left(-2 \frac{\Delta_{t, m}}{\delta_{t}^{2}}\right) \\
& \times \exp \left(-\frac{\Delta_{n, m}}{\delta_{n}}\right) \exp \left(-\frac{\Delta_{t, m}^{2}}{\delta_{t}^{2}}\right)
\end{aligned}
$$

are used to construct the material tangent operator. In case of unloading and reloading, there might be discontinuities in the traction-opening relations whenever the reloading direction differs from the unloading direction, see [26]. To prevent such artificial jumps, the tractions are defined as:

$t_{n}^{u n l}=\frac{\Delta_{\text {eff }}}{\Delta_{\max }} t_{n}\left(\Delta_{n, m}^{*}, \Delta_{t, m}^{*}\right)$
$t_{t}^{u n l}=\frac{\Delta_{\text {eff }}}{\Delta_{\max }} t_{t}\left(\Delta_{n, m}^{*}, \Delta_{t, m}^{*}\right)$

where

$\Delta_{n, m}^{*}=\frac{\Delta_{\text {max }}}{\Delta_{\text {eff }}} \Delta_{n}$
$\Delta_{t, m}^{*}=\frac{\Delta_{\text {max }}}{\Delta_{\text {eff }}} \Delta_{t}$

and $\Delta_{\max }$ is the maximum effective opening reached during the history and $\Delta_{\text {eff }}$ is the current effective opening, both based on mechanical openings. The corresponding derivatives are:

$$
\begin{aligned}
\frac{\partial t_{n}}{\partial \Delta_{n, m}}= & \frac{\partial t_{n}}{\partial \Delta_{n, m}^{*}}+\frac{\Delta_{n, m}}{\Delta_{\max } \Delta_{\mathrm{eff}}} t_{n}-\frac{\Delta_{n, m}^{2}}{\Delta_{\mathrm{eff}}^{2}} \frac{\partial t_{n}}{\partial \Delta_{n, m}^{*}} \\
& -\frac{\Delta_{n, m} \Delta_{t, m}}{\Delta_{\mathrm{eff}}^{2}} \frac{\partial t_{n}}{\partial \Delta_{t, m}^{*}} \\
\frac{\partial t_{n}}{\partial \Delta_{t, m}}= & \frac{\partial t_{n}}{\partial \Delta_{t, m}^{*}}+\beta^{2} \frac{\Delta_{t, m}}{\Delta_{\max } \Delta_{\mathrm{eff}}} t_{n}-\beta^{2} \frac{\Delta_{t, m}^{2}}{\Delta_{\mathrm{eff}}^{2}} \frac{\partial t_{n}}{\partial \Delta_{t, m}^{*}} \\
& -\beta^{2} \frac{\Delta_{n, m} \Delta_{t, m}}{\Delta_{\mathrm{eff}}^{2}} \frac{\partial t_{n}}{\partial \Delta_{n, m}^{*}} \\
\frac{\partial t_{t}}{\partial \Delta_{t, m}}= & \frac{\partial t_{t}}{\partial \Delta_{t, m}^{*}}+\beta^{2} \frac{\Delta_{t, m}}{\Delta_{\max } \Delta_{\mathrm{eff}}} t_{t}-\beta^{2} \frac{\Delta_{t, m}^{2}}{\Delta_{\mathrm{eff}}^{2}} \frac{\partial t_{t}}{\partial \Delta_{t, m}^{*}} \\
& -\beta^{2} \frac{\Delta_{n, m} \Delta_{t, m}}{\Delta_{\mathrm{eff}}^{2}} \frac{\partial t_{t}}{\partial \Delta_{n, m}^{*}} \\
\frac{\partial t_{t}}{\partial \Delta_{n, m}}= & \frac{\partial t_{t}}{\partial \Delta_{n, m}^{*}}+\frac{\Delta_{n, m}}{\Delta_{\max } \Delta_{\mathrm{eff}}} t_{t}-\frac{\Delta_{n, m}^{2}}{\Delta_{\mathrm{eff}}^{2}} \frac{\partial t_{t}}{\partial \Delta_{n, m}^{*}} \\
& -\frac{\Delta_{n, m} \Delta_{t, m}}{\Delta_{\mathrm{eff}}^{2}} \frac{\partial t_{t}}{\partial \Delta_{t}^{*}}
\end{aligned}
$$

The expression for $\frac{\partial t_{t}}{\partial \Delta_{t, m}^{*}}$ and the other comparable terms are identical to the corresponding expressions given above 
provided that, for example $\Delta_{t, m}$ and $\Delta_{n, m}$ are replaced by $\Delta_{t, m}^{*}$ and $\Delta_{n, m}^{*}$.

The linearization of the thermal equilibrium requires the sensitivity of the interface heat flux with respect to temperature jump. In case loading and unloading, the derivative,

$$
\begin{aligned}
\frac{\partial q_{i}}{\partial \Delta \theta}= & \frac{1}{2} \frac{1}{\sqrt{q_{n}^{2}+q_{t}^{2}}}\left(2 q_{n}\left((1-d) k_{s}+k_{g}\right) \mathbf{m} \cdot \mathbf{n}\right. \\
& \left.+2 q_{t}(1-d) k_{s} \mathbf{m} \cdot \mathbf{t}\right)
\end{aligned}
$$

is used in the solution of the linearized thermal equilibrium equations. In case of contact, the derivative takes the following form,

$$
\frac{\partial q_{i}}{\partial \Delta \theta}=\frac{1}{2} \frac{1}{q_{n}}\left(2 q_{n}\left(\left(1-d_{c}\right) k_{s}\right) \mathbf{m} \cdot \mathbf{n}\right)
$$

\section{References}

1. van den Bosch M, Schreurs PJG, Geers MGD (2008) On the development of a 3D cohesive zone element in the presence of large deformations. Comput Mech 42(2):171-180

2. Chandra N, Li H, Shet C, Ghonem H (2002) Some issues in the application of cohesive zone models for metal-ceramic interfaces. Int J Solids Struct 39:2817-2825

3. Xu X-P, Needleman A (1993) Void nucleation by inclusion debonding in a crytal matrix. Model Simulat Mater Sci Eng 1:111-132

4. Willam K, Rhee I, Xi Y (2003) Thermal degradation of heterogeneous concrete materials. ASCE J Mater Civil Eng 17:276-285

5. Hattiangadi A, Siegmund T (2005) An analysis of the delamination of an environmental protection coating under cyclic heat loads. Eur J Mech A Solids 24:361-370

6. Willam K, Rhee I, Shing B (2004) Interface damage model for thermomechanical degradation of heterogeneous materials. Comput Methods Appl Mech Eng 193:3327-3350

7. Steinmann P, Häsner $\mathrm{O}(2005)$ On material interfaces in thermomechanical solids. Arch Appl Mech 75:31-41

8. Krol O (2007) Thermo-mechanical modeling of solids and interfaces. PhD thesis, Technische Universität Kaiserslautern

9. Fagerström F, Larsson R (2008) A thermo-mechanical cohesive zone formulation for ductile fracture. J Mech Phys Solids 56:30373058

10. Hattiangadi A, Siegmund T (2004) A thermomechanical cohesive zone model for bridged delamination cracks. J Mech Phys Solids 52:533-566
11. Hattiangadi A, Siegmund T (2005) A numerical study on interface crack growth under heat flux loading. Int J Solids Struct 42:63356355

12. Tzou DY (1990) The singular behaviour of the temperature gradient in the vicinity of a macrocrack tip. Int J Heat Mass Transf 33:2625-2630

13. Zavarise G, Wriggers P, Stein E, Schrefler BA (1992) Real contact mechanisms and finite element formulation: a coupled thermomechanical approach. Int J Numer Methods Eng 35:767-785

14. Ciavarella M, Greenwood JA, Paggi M (2008) Inclusion of 'interaction' in the Greenwood and Williamson contact theory. Wear 265:729-734

15. Ciavarella M, Dibello S, Demelio G (2008) Conductance of rough random profiles. Int J Solids Struct 45:879-893

16. Barber JR (2003) Bounds on the electrical resistance between contacting elastic rough bodies. Proc R Soc Lond A 459:53-66

17. Borri-Brunetto M, Carpinteri A, Invernizzi S, Paggi M (2006) Micro-slip of rough surfaces under cyclic tangential loading. In: Wriggers P, Nackenhorst U (eds) Analysis and simulation of contact problems. Lecture Notes in Applied and Computational Mechanics, vol 27, pp 333-340

18. Camacho GT, Ortiz M (1996) Computational modelling of impact damage in brittle materials. Int J Solids Struct 33:2899-2938

19. Margolin LG (1984) A generalized Griffith criterion for crack propagation. Eng Fract Mech 19:539-543

20. Dienes JK (1986) Comments on 'A generalized Griffith criterion for crack propagation'. Eng Fract Mech 23:615-617

21. Paggi M, Carpinteri A, Zavarise G (2006) A unified interface constitutive law for the study of fracture and contact problems in heterogeneous materials. In: Wriggers P, Nackenhorst U (eds) Analysis and simulation of contact problems. Lecture Notes in Applied and Computational Mechanics, vol 27, pp 297-304

22. Carpinteri A, Paggi M, Zavarise G (2008) The effect of contact on the decohesion of laminated beams with multiple micro-cracks. Int J Solids Struct 45:129-143

23. Van den Bosch M, Schreurs PJG, Geers MGD (2006) An improved description of the exponential $\mathrm{Xu}$ and Needleman cohesive zone law for mixed-mode decohesion. Eng Fract Mech 73:1220-1234

24. Swain M (ed) (1994) Structure and properties of ceramics. Mater Sci Technol 11:VCH

25. Espinoza DH, Zavattieri PD (2003) A grain level model for the study of failure initiation and evolution in polycrystalline brittle materials. Part II: Numerical examples. Mech Mater 35:365-394

26. Kregting R (2005) Cohesive zone models: towards a robust implementation of irreversible behaviour. Department of Mechanical Engineering, Eindhoven University of Technology, Internal Report. www.mate.tue.nl/mate/showabstract.php/5169 